\title{
La perspectiva de alfabetización freireana y el enfoque didáctico constructivista con base en las investigaciones psicogenéticas: continuidades y rupturas para pensar los procesos de alfabetización inicial en personas jóvenes y adultas
}

Marcela Kurlat

Universidad de Buenos Aires (Argentina).

marcelakurlat@yahoo.com.ar

Fecha de recepción: 21 de febrero de 2020.

Fecha de aceptación: 30 de abril de 2020.

\begin{abstract}
Resumen
El presente artículo se propone analizar continuidades y rupturas entre la perspectiva de alfabetización freireana y el enfoque didáctico constructivista con base en las investigaciones psicogenéticas, a modo de insumo para pensar los procesos de alfabetización inicial en personas jóvenes y adultas. Desde un devenir de catorce años de investigación en los que hemos intentado conocer la especificidad de la construcción del sistema de escritura en dicha población, hemos entrado en diálogo y reflexión con los aportes freireanos a los procesos de alfabetización de personas jóvenes y adultas desde la educación popular, realizando desde la praxis de docencia e investigación procesos comparativos con el enfoque constructivista de base psicogenética. Sostenemos la convergencia de ambos enfoques desde una mirada político-pedagógica y la superación didáctica del enfoque constructivista, desde una enseñanza de la lectura y la escritura capaz de materializar con más potencia aun que los propios postulados del método freireano, una alfabetización liberadora y crítica.
\end{abstract}

Palabras clave: alfabetización de personas jóvenes y adultas, perspectiva freireana, enfoque didáctico constructivista, investigaciones psicogenéticas, continuidades y rupturas.

Freirean literacy perspective and constructivism didactic approach focuses in psychogenetic research: continuities and breaks for inicial youth and adults literacy processes reflection

\begin{abstract}
This article inteds to analyze continuities and ruptures between the literacy freirean perspective and constructivism didactic approach, based in psychogenetic research, as contribution for youth and adults literacy processes reflection. From a future of fourteen year research we have tried to know the specificity of written sistem construction, we have entered into dialogue with freirian contributions, working in comparative
\end{abstract}


processes from both perspectives. We hold the convergence of both approaches from a pedagogical political perspective, and the didactic improvement of the constructivism approach. The teaching of reading and writting from this perspective materializes even more power than the freirean postulates themselves, a liberating and critical literacy.

Keywords: youth and adults literacy, freirean perspective, constructivism didactic approach, psychogenetic research, continuities and ruptures

\section{Introducción}

Con frecuencia, alfabetizadores y alfabetizadoras de personas jóvenes y adultas plantean dificultades para pensar la enseñanza frente a estudiantes que aún no leen ni escriben convencionalmente. "Hace años que vienen las señoras y no avanzan nada, ya no sé qué hacer" "Necesito ayuda, no sé cómo alfabetizar a 'analfabetos puros" "Me produce mucha frustración, no me formaron para enseñar a leer y escribir a esta población" "Hay cosas que no puedo entender... trabajamos con la palabra generadora de Freire, pero no nos alcanza", son frases que solemos escuchar en cursos, seminarios y espacios de formación de alfabetizadores y alfabetizadoras. En los últimos catorce años, desde el "Programa de Desarrollo Sociocultural y Educación Permanente: la educación de jóvenes y adultos más allá de la escuela” (dirigido por la Dra. Sirvent, con sede en el IICE-UBA), nos hemos abocado a indagar los factores involucrados en la construcción del sistema de escritura en personas jóvenes y adultas, en relación con los procesos de construcción de demandas individuales y colectivas por aprendizajes permanentes, tanto en espacios de terminalidad de la escuela primaria como en aquellos de alfabetización de movimientos sociales. Buscamos generar conocimiento acerca de la especificidad de la alfabetización en esta población desde una perspectiva psicogenética, es decir, intentando capturar el punto de vista del sujeto que aprende a leer y escribir, las hipótesis y niveles de conceptualización que construye en su proceso de apropiación de la escritura, así como las intervenciones didácticas que promueven dicha apropiación, considerando su dimensión político-pedagógica y psicosocial.

En este devenir, hemos entrado en diálogo y reflexión con los aportes freireanos a los procesos de alfabetización de personas jóvenes y adultas desde la educación popular, realizando desde la praxis de docencia e investigación procesos comparativos con el enfoque constructivista de base psicogenética, cuya referente máxima en el dominio lingüístico ha sido la Dra. Emilia Ferreiro. Las propias investigaciones en el área con foco en la población joven y adulta (Kurlat, 2011, 2014, 2016; Kurlat y Perelman, 2012; Kurlat y Chichizola, 2017; Kurlat, Chichizola y Risso, en prensa), han dado origen a un corpus teórico-empírico que permite establecer continuidades y rupturas entre ambas perspectivas y propuestas para pensar los procesos de alfabetización inicial en esta población específica. A ello nos abocaremos en el presente artículo, describiendo cada una de las propuestas en sendos apartados, para luego compartir hallazgos propios en el área y finalizar con la comparación entre ambas perspectivas y la identificación de dichas continuidades y rupturas. Dado que el presente número de la Revista del IICE está dedicado a Paulo Freire, con numerosísimas contribuciones sobre su obra político-pedagógica más amplia, aquí nos focalizaremos exclusivamente en su propuesta de alfabetización, dando por conocido su anclaje mayor.

\section{El método freireano de alfabetización}

En La educación como práctica de la libertad (2004), Freire defiende una educación que coloque a las personas en diálogo constante, que las predisponga a sucesivas 
revisiones, a análisis críticos de sus descubrimientos y a una "cierta rebeldía". En este sentido:Pensábamos en una alfabetización directa y realmente ligada a la democratización de la cultura, que fuese una introducción a esta democratización. Una alfabetización que, por eso mismo, no considerase al hombre espectador del proceso, cuya única virtud es tener paciencia para soportar el abismo entre su experiencia existencial y el contenido que se le ofrece para su aprendizaje sino que lo considerase como sujeto.

En verdad, solamente con mucha paciencia es posible tolerar, después de las durezas de un día de trabajo o de un día sin trabajo, lecciones que hablen de ala, Pedro vio el ala, El ala es del ave, lecciones que hablan de Evas y uvas a los hombres que a veces conocen pocas Evas y nunca comerán uvas: Eva vio la uva. Pensábamos en una alfabetización que fuese en sí un acto de creación capaz de desencadenar otros actos creadores, en una alfabetización en que el hombre, no siendo su objeto, desarrolle la impaciencia, la vivacidad, característica de los estados de estudio, la invención, la reivindicación. (Freire, 2004: 65)

Así, halló la respuesta en la construcción de "un método activo, dialogal y participante" (Freire, 1976, 2004), un método que caracterizó como "crítico y de espíritu crítico"; que conllevaba una modificación del programa educacional y el uso de técnicas que denominó de reducción y codificación, Para introducir el concepto de cultura, al mismo tiempo gnoseológica y antropológicamente, hicimos la reducción de este concepto a trazos fundamentales, once situaciones existenciales codificadas capaces de desafiar a los grupos y hacérselas comprender por medio de su descodificación. (Freire, 2004: 70)

Las fases de elaboración y de acción práctica del método fueron descritas de la siguiente manera (Freire, 1984, 2004):

1. Obtención del universo vocabular de los grupos con los cuales se trabajará: este estudio se hace a través de encuentros informales con los moradores del área a alfabetizar. Se obtienen los vocablos con sentido existencial, con mayor contenido emocional, así como aquellas expresiones típicas del pueblo.

2. Selección del universo vocabular estudiado, según ciertos criterios: riqueza fonética, dificultades fonéticas de la lengua, colocadas en secuencias que van gradualmente de las dificultades menores a las mayores; y tenor pragmático de las palabras, que implica su compromiso con la realidad social, política y cultural dada.

3. Creación de situaciones existenciales típicas del grupo con el que se va a trabajar: situaciones problema codificadas, que incluyen elementos que serán decodificados por los grupos, con la colaboración del coordinador. Debates en los que se analizan problemáticas, en las que se colocan vocablos generadores, en grados según sus dificultades fonéticas.

4. Elaboración de fichas desde la descomposición de las familias fonéticas que corresponden a los vocablos generadores.

5. Una vez que se agota el análisis oral de la situación dada (a través de una lámina), lo que se denomina descodificación, el educador/a pasa a la visualización de la palabra generadora; para la visualización y no para la memorización. Luego de esto, y una vez establecido el vínculo semántico entre ella y el objeto a que se refiere, se ofrece al educando/a un cartel donde aparece la palabra sin el objeto nombrado. Luego, se presenta la misma separada en sílabas para pasar después a la visualización de las familias fonémicas que componen la palabra de estudio. Estas familias, que son estudiadas 
aisladamente, se presentan luego en conjunto, para llegar al reconocimiento de las vocales en último lugar.

Ejemplo:

1. Se comparte una lámina con una situación de construcción. Se presenta la palabra tijolo ("ladrillo"), como primera palabra generadora. Se discute la situación presentada.

2. Luego se muestra la palabra sin la imagen: TIJOLO.

3. Se visualizan sus "trozos": TI - JO - LO y se reconocen las familias fonémicas (ta-teti-to-tu, ja-je-ji-jo-ju, la-le-li-lo-lu).

Se comparan las sílabas y se realizan ejercicios de lectura para su fijación.

4. Se crean palabras nuevas combinando sílabas (tatu, lajota, loja, jato, lote, tela).

Se afirma así que "Esa noche el hombre empieza a escribir", clasificando el trabajo como método ecléctico analítico-sintético, que da centralidad al descubrimiento del mecanismo de las combinaciones fonémicas. El método propone una primera etapa de alfabetización, educación problematizadora o educación de la comunicación, que busca e investiga la "palabra generadora", y luego una segunda etapa, de "posalfabetización", que busca e investiga un "tema generador" (Freire, 1970: 132).

Para Giroux (en Freire y Macedo, 1989), la alfabetización es fundamentalmente para Freire un proyecto político por el cual los hombres y las mujeres sostienen su derecho y su responsabilidad no solo a leer, comprender y transformar sus propias experiencias, sino también a reconstituir su relación con la sociedad toda: leer no consiste solamente en decodificar la palabra o el lenguaje escrito; antes bien, es un acto precedido por (y entrelazado con) el conocimiento de la realidad. El lenguaje y la realidad están interconectados dinámicamente. La comprensión que se alcanza a través de la lectura crítica de un texto implica percibir la relación que existe entre el texto y el contexto. (Freire y Macedo, 1989: 51)

Si bien Freire se negaba a reducir la alfabetización a la "pura enseñanza de la palabra, de las sílabas o de las letras", o a la "memorización mecánica de las sílabas ba-be-bi-bo-bu", su método no pudo sortear la dicotomía entre la "palabra generadora" y la acción de los sujetos: estudios revelan que los saberes previos vinculados al sistema de escritura no se retomaban en los espacios educativos, centrándose las prácticas alfabetizadoras en el desmembramiento y unión de sílabas, alejadas de los postulados sobre la escritura como acto de creación y conocimiento (Aravedo Reséndiz, 2007; Oliveira, 1988, en Lorenzatti, 2009). La propuesta de alfabetización freireana continúa formando parte de los llamados métodos analíticos, que parten de unidades con significado (palabras o frases), que se descomponen progresivamente hasta alcanzar unidades menores. Más allá de la concepción de sujeto político freireano, de la riqueza de los círculos de cultura como espacios de concienciación, del diálogo como objetivación de la propia realidad y de la propuesta de lectura del mundo para la transformación de la propia realidad, el método de alfabetización en su interpretación didáctica continúa ignorando las funciones comunicativas de la lengua, dando centralidad a la enseñanza de sílabas y fonemas e ignorando los caminos de escritura - en clave psicogenética- de las personas que se alfabetizan.

Si bien desde nuestra perspectiva acordamos con la dimensión sociopolítica de la perspectiva freireana, consideramos necesario profundizar en las dimensiones lingüísticas y cognitivas de los procesos de apropiación del sistema de escritura por parte de los 
sujetos jóvenes y adultos, considerar los procesos psicogenéticos y didácticos involucrados en dicho aprendizaje. Describiremos estos aspectos en el próximo apartado.

\section{El enfoque constructivista sobre los procesos de apropiación del sistema de escritura por parte de los sujetos}

Desde finales de los años 70 se han desarrollado numerosas investigaciones desde la perspectiva psicogenética sobre la construcción del sistema de escritura por parte de niños/as en diferentes países (Ferreiro y Teberosky, 1979; Teberosky, 1982; Ferreiro y Gómez Palacio, 1982; Tolchinsky y Levin, 1982; Ferreiro, 1986, 1994; Kaufman, 1988; Vernon, 1991, 1997, 2004; Quinteros, 1997, 2004; Molinari, 2007; Vernon y Cano, 2008; Zamudio, 2008; Grunfeld, 2012). Estas investigaciones han dado un giro a la interpretación de la escritura como un sistema de representación que requiere ser comprendido por los sujetos, y han contribuido a echar luz sobre los procesos constructivos que se ponen en juego en la apropiación del sistema de escritura como un nuevo objeto de conocimiento, con la intención de entender la evolución de los sistemas de ideas que las personas elaboran sobre la naturaleza de ese objeto social. Sus descubrimientos acerca de la psicogénesis de la lengua escrita han puesto en evidencia la progresión regular en su modo de conceptualizar la lengua escrita, que va de etapas presilábicas al reconocimiento de principios alfabéticos en nuestras escrituras. Dichas autoras han denunciado las dificultades que aparecen en las aulas al predominar la visión que educadores/as como adultos/as ya alfabetizados/as poseen sobre el sistema de escritura ( $\sin$ partir de las conceptualizaciones que quienes aprenden tienen sobre el mismo); la confusión existente entre el escribir y el dibujar letras, y la reducción del conocimiento del lector al conocimiento de las letras y su valor sonoro convencional. Ello conlleva la generación de propuestas didácticas de descifrado, copia, memorización y repetición, considerándose la escritura como un código, una técnica de transcripción. El desconocimiento del proceso de aprendizaje de este sistema ha sido un factor de fracaso escolara lo largo de la historia.

En 1983, Emilia Ferreiro demostró la existencia de conceptualizaciones semejantes y diferentes en las poblaciones infantil y adulta, reencontrando las mismas respuestas en relación a: el análisis de las partes del nombre propio; las formas de escritura presilábicas, silábicas, silábico-alfabéticas y alfabéticas; la manifestación de los mismos requerimientos de hipótesis de cantidad mínima y variedad interna; la similar distinción entre "lo que está escrito" y "lo que se puede leer"; así como dificultades parecidas para manejar las relaciones entre el todo y las partes a nivel de la palabra escrita. Las mayores diferencias entre ambas poblaciones son: la ausencia en las personas jóvenes y adultas de los niveles de respuesta más primitivos presentes en la población infantil; la existencia de una fuerte distinción entre las grafías letras y las grafías números en personas adultas; una comprensión de las segmentaciones del texto casi ausente en la población infantil; una mayor comprensión de las funciones sociales de la lengua escrita en la población adulta.

En este marco, se considera que en las sociedades letradas las personas estamos inmersas en la cultura escrita desde el nacimiento y hasta la muerte. Esto quiere decir que, siguiendo los criterios de continuidad, simultaneidad de propuestas y progresión, la alfabetización se desarrolla a lo largo de todos los niveles educativos y está determinada por diversos procesos culturales y circunstancias personales, se da tanto dentro como fuera de la escuela, es "más que leer y escribir", involucra prácticas culturales y relaciones sociales, usos y conocimientos que promueven la participación de los sujetos en las sociedades letradas. Leer es comprender, construir sentido entre lo que el texto dice y lo que el lector le aporta al texto. No es decodificar la letra impresa (o en 
el caso del escritor, dibujarla o tipearla correctamente) sino algo mucho más complejo y profundo: significa construir sentido, ya que el lenguaje escrito es un sistema de representación, no un código. Leer hace posible la integración en una comunidad de lectores y escritores, y la apropiación paulatina de las prácticas sociales del lenguaje. Formarse como escritor, como plantea Kaufman (2007), no es "saber las letras y sus sonidos" — ni familias de sílabas, podríamos agregar-, sino que implica apropiarse de la lengua escrita de una sociedad, conocer qué representan las marcas gráficas, participar en prácticas en las que se aprenden, simultáneamente, cuestiones vinculadas con el lenguaje escrito (características de los géneros a los que pertenecen los textos que circulan socialmente, cuestiones de coherencia textual, entre otras) y el sistema de escritura (las letras, otros signos y las relaciones entre ellos). Algunos modos de leer son anteriores a la lectura convencional y constituyen pasos previos para ser un lector. Dejar a la persona que aprende únicamente con la información de las familias silábicas y sus combinaciones le permite sonorizar, pero no leer ni escribir. Para construir el significado de un texto se ponen en juego variadas estrategias que van más allá del mero conocimiento de las letras y los sonidos. Los sujetos necesitan interactuar con la lengua escrita, sumergirse en prácticas de lectura y escritura contextualizadas, necesitan pensar la escritura y hacer uso de ella para la reflexión. Desde allí se concibe la enseñanza en un enfoque constructivista, que a lo largo de sucesivas décadas se ha sustentado en investigaciones didácticas específicas, mayormente en el campo infantil en contextos escolares (Lerner, 1987, 2001; Kaufman, 1988; Ferreiro, 1989; Kaufman, Castedo, Teruggi y Molinari, 1989; Teberosky, 1992; Tolchinsky, 1993; Weisz, 1996; Nemirosky, 1999; Castedo, 2010, y citadas en Grunfeld, 2012).

\section{Investigaciones propias sobre los procesos de alfabetización de personas jóvenes y adultas: caminos y laberintos de escritura}

Como ya se ha adelantado en la introducción, numerosos proyectos de extensión universitaria y de investigación ${ }^{1}$ de los últimos catorce años han podido identificar caminos de escritura propios de las personas jóvenes y adultas que aprenden a leer y a escribir, así como cierta trama de procesos psicosociales y didácticos involucrados en la alfabetización de esta población. Las propias investigaciones describen posibles caminos de escritura que transita toda persona que aprende a leer y escribir, invitan a pensar los procesos de alfabetización de personas jóvenes y adultas como una trenza de tres hebras: los niveles de conceptualización sobre el sistema de escritura convergentes con los conocidos en la población infantil como primera hebra, cuyos caminos de construcción muchas veces aparecen laberínticos, anudados, entrecruzados por las siguientes dos hebras de dicho proceso: las "marcas de exclusión" y las "marcas de enseñanza" (Kurlat, 2011; Kurlat y Perelman, 2012). Las tres hebras visibilizan la compleja urdimbre y trama de procesos psicosociales y didácticos involucrados en los caminos de apropiación del sistema de escritura (Kurlat, 2014; 2016), fundamentales

1 Hacemos referencia a los siguientes proyectos y cátedras: - "Procesos de enseñanza y aprendizaje de la lectura y la escritura en el aula de jóvenes y adultos, estudio comparativo de casos", Subproyecto del Proyecto UBACyT Foo6, dirigido por la Dra. Sirvent., con subsidio del Centro de Cooperación Regional para la Educación de Adultos en América Latina y el Caribe (CREFAL) durante el período 2006-2008. La dirección del subproyecto estuvo a cargo de la Prof. Amanda Toubes. -Proyecto UBANEX “Problemas y necesidades en la formación de educadores de jóvenes y adultos”, aprobado por Resolución (CD) № 2450, 2011. Dirección: Profs. Amanda Toubes e Hilda Santos. "Procesos de construcción del sistema de escritura en el aula de adultos. Estudio de casos”, Tesis de Maestría de Marcela Kurlat en Psicología Educacional por la Facultad de Psicología, Universidad de Buenos Aires. Dirección: Dra. Flora Perelman, con beca de maestría dirigida por la Dra. Sirvent, aprobada en 2014. - "Procesos psicogenéticos, psicosociales y didácticos en la alfabetización inicial de personas jóvenes y adultas. Estudio de caso", Tesis de Doctorado en Educación por la Facultad de Filosofía y Letras, Universidad de Buenos Aires de Marcela Kurlat. Dirección: Dras. Sirvent y Perelman, entregada para su evaluación en 2015.-Cátedra de Educación de Adultos, Facultad de Filosofía y Letras, Universidad de Buenos Aires, carrera de Ciencias de la Educación, coordinada por la Prof. Amanda Toubes. La misma ha propuesto al PAEByT como espacio de aprendizaje de sus estudiantes, a modo de acercamiento al campo. 
para sustentar propuestas de alfabetización y de formación de educadores y educadoras en el área, que retoman el enfoque didáctico constructivista cuyo objeto de enseñanza son las prácticas sociales del lenguaje: leer, escribir, hablar y escuchar (Castedo, 2010).

Toda persona que llega a centros de alfabetización ha construido ideas, hipótesis, conceptualizaciones sobre lo que la escritura representa, haya o no ido a la escuela previamente. Toda persona llega a los centros con un camino de escritura particular que debemos conocer y respetar, a partir del cual se trabaja para promover la apropiación progresiva del sistema de escritura y de la lengua escrita como objetos de conocimiento.

Es necesario seguir la lógica de pensamiento de las personas jóvenes y adultas en proceso de alfabetización, recuperar lo que sí conocen, cuáles son sus estrategias lectoras, cuáles los textos de circulación social con los que interactúan en la vida cotidiana, cuáles sus ideas acerca de lo que la escritura representa y ofrecerles situaciones con sentido para propiciar y potenciar la alfabetización, e ir encontrando el hilo de Ariadna que ayude a salir de los laberintos de escritura y de enseñanza que suelen explicitar los/as alfabetizadores/as.

Veamos algunos ejemplos:

\begin{tabular}{lll}
\hline NIVEL DE & ESCRITURAS & COMENTARIOS E INTERVENCIONES NECESARIAS \\
CONCEPTUALIZACIÓN &
\end{tabular}

PRESILÁBICO O PREFONETIZANTE
Alejandro, de más de 30 años, escribe:

ALJANDROE (Alejandro) ALJANEDRO (harina) ALJRADEO (azúcar) ALJANDRO (levadura) ALJANERO (agua) ALJRADNO (leche) ALJARDEO (crema) (basado en las letras de su nombre), con hipótesis de cantidad (más de 5 marcas gráficas por escritura) y de variedad intra e interfigural (varía el orden de las marcas gráficas para que "digan” algo diferente en cada texto).

Su escritura e interpretación es continua (señalando con su dedo cada escritura y verbalizando la palabra completa que quiso escribir, sin análisis de partes).

Alejandro necesitará que lo acompañemos desde intervenciones que lo lleven a fonetizar la escritura, a comenzar a establecer relaciones entre la oralidad y la escritura, a ingresar en un nivel silábico desde la identificación de índices cualitativos y cuantitativos de los textos, fundamentalmente desde la apelación a textos significativos que se conocen de memoria, como el nombre propio, los días de la semana, nombres de familiares, y realizar preguntas tales como: “¿dónde dice?”, “¿cómo te das cuenta?”, "mirá, empieza como tu nombre”.

SILÁBICO Ramón, de más de 40 años, escribe:

Ramón adjudica una marca gráfica por sílaba, con valor sonoro convencional (es decir, coloca una de las letras que efectivamente corresponde con la escritura que quiere producir, con valor silábico). Para él, dos marcas gráficas son suficientes para que un texto "pueda decir" y no le provoca conflicto la variedad interfigural, por lo que una intervención posible podría ser: “¿te parece que Angi y David se escribirán igual (Al), o para que digan distinto, habrá que agregar alguna letra más a alguno de los nombres?".

$\begin{array}{lll}\text { SILÁBICO-ALFABÉTICO } & \text { Patricia, de } 35 \text { años escribe: } & \text { Patricia oscila entre adjudicar valor silábico o fonémico a las } \\ & & \text { marcas gráficas que produce. Para ella, una intervención posible } \\ & \text { AINA (harina) } & \text { puede ser el trabajo con letras móviles: “te doy todas las que van, } \\ \text { AUO (azúcar) } & \text { no falta ni sobra ninguna, ¿en qué orden te parece que van para } \\ \text { EMDUA (levadura) } & \text { que diga...?”. También la interpretación y producción por partes de } \\ \text { AUA (agua) } & \text { la palabra ayuda a que la persona se dé cuenta si faltan o no letras. }\end{array}$




\begin{tabular}{lll}
\hline $\begin{array}{l}\text { NIVEL DE } \\
\text { CONCEPTUALIZACIÓN }\end{array}$ & ESCRITURAS & COMENTARIOS E INTERVENCIONES NECESARIAS \\
\hline ALFABÉTICO INICIAL & Paola, de 20 años, escribe: & $\begin{array}{l}\text { Paola ya se apropió de la alfabeticidad del sistema, pero aún } \\
\text { transforma las sílabas complejas en sílabas directas simples } \\
\text { consonante-vocal. Una intervención posible es pedirle que }\end{array}$ \\
& HARINA & $\begin{array}{l}\text { interprete la propia producción: “¿cómo leés azúcar? Mostrame } \\
\text { con tu dedo. ¿Con cuál termina azúcar?”, así como darle }\end{array}$ \\
& AGUA & información que aporte a repensar su propia escritura: “leche se \\
& AZUCA & escribe con la misma de chocolate, de chancho”, o “te escribo la \\
LEHE (leche) & palabra CREMOSO, a ver qué parte te sirve para escribir CREMA”. \\
\hline
\end{tabular}

Cada intervención se ajusta a la persona, a lo que a ella la pueda ayudar a avanzar desde su escritura singular. Al mismo tiempo, para acompañar e intervenir didácticamente desde estos caminos de escritura diversos que encontramos en las personas que aprenden a leer y escribir, nos posicionamos en una perspectiva de enseñanza desde cuatro situaciones didácticas fundamentales, ya presentes en los diseños curriculares de la escuela primaria infantil: la lectura y escritura a través del alfabetizador/a, la lectura y escritura por sí misma de la persona que aprende. Comenzamos a probar y a analizar intervenciones didácticas desde el enfoque constructivista que resultan fértiles para promover el avance conceptual y que ya han sido validadas por numerosas investigaciones en la población infantil, ahora también en la población joven y adulta. Dichas intervenciones se orientan a reflexionar sobre las relaciones entre oralidad y escritura.

En este sentido, como intervenciones fértiles en los procesos de escritura, potenciadoras del avance conceptual, hemos identificado en la escritura de la persona por sí misma:

» la anticipación de cuántas letras harán falta para escribir (“¿Cuántas necesitarás para escribir?"),

» la interpretación de la propia producción (“¿Cómo lo leés vos?”),

» la revisión de la propia escritura, la interpretación de las partes de la escritura, la lectura de control y la lectura con detención (“¿Hasta ahí qué dice, qué escribiste?”, “¿Ya dice o falta?”),

» el uso de palabras referentes para la escritura de nuevas palabras, la construcción de un ambiente alfabetizador con textos seguros que permitan ser fuente de nuevas escrituras (“¿Qué parte de esta palabra te puede servir para escribir la nueva?”),

» la verbalización y escritura de palabras para comparar vínculos entre oralidad y escritura,

» el dar siempre información que permita comparar y agrupar una palabra que empieza como otras, reflexionando acerca del inicio sonoro y la letra correspondiente.

Desde procesos de lectura, las intervenciones fértiles identificadas hasta el momento han sido:

» un texto a partir de índices cualitativos o cuantitativos en coordinación con información verbal (“¿Dónde dice?” lo que se sabe que dice),

» el pedido de justificación desde las marcas del texto (“¿Cómo te das cuenta?”, “¿Por qué te parece?"),

» la localización de un texto a partir de la vinculación entre la linealidad en la lectura y la linealidad en la escritura, la lectura del adulto para la localización de un enunciado escrito en función del orden en la emisión oral,

» el ingreso al universo de sentido de los textos, que permite hacerse preguntas acerca de lo que la escritura representa, atender a los índices internos de los textos en coordinación con la información verbal. 
En síntesis, estas intervenciones implican: la construcción de un ambiente alfabetizador con palabras conocidas y seguras, a partir de las cuales interpretar, analizar y producir nuevos textos; promover reflexiones sobre las relaciones entre oralidad y escritura desde proyectos didácticos contextualizados, con funciones comunicativas reales, sociales y significativas, conociendo y acompañando los caminos de escritura de las personas que aprenden desde intervenciones que las ayuden a avanzar hacia la fonetización de la escritura, desde el conocimiento progresivo del lenguaje que se escribe.

Veamos entonces algunas dimensiones de análisis comparativo con el método freireano de alfabetización en el próximo apartado.

\section{Acercamientos y distancias con el método de alfabetización freireano}

Paulo Freire, a lo largo de sus desarrollos teóricos, contribuyó a redefinir la alfabetización en un sentido multidimensional, como acto político, acto de conocimiento, acto creador, acto de lectura de la palabra y la realidad, rompiendo con la concepción tradicional de la educación y del sujeto cognoscente. La perspectiva de alfabetización que hemos desarrollado en el apartado anterior, coincide con los siguientes postulados freireanos (1998):

» La alfabetización de personas jóvenes y adultas constituye un acto político, un acto de conocimiento, un acto creador.

» La lectura del mundo precede a la lectura de la palabra y la lectura de esta implica la continuidad de la lectura de aquel, en un movimiento del mundo a la palabra y de la palabra al mundo.

》 La comprensión del texto implica la de las relaciones entre el texto y el contexto.

" El aprendizaje de la lectura conlleva la significación profunda de los textos que se abordan.

Asumimos que la alfabetización hace referencia a la red compleja de conocimientos que los sujetos necesitan para desenvolverse en una sociedad letrada desde un análisis crítico del entorno cotidiano (Sirvent, 2005; Buitron, Cruciani et al., 2008); acordamos con Freire en que el proceso de alfabetización implica la "lectura de la palabra" como constitutiva de la "lectura de la realidad", y al mismo tiempo sostenemos, retomando la perspectiva de Ferreiro, que la alfabetización es un proceso de apropiación de la lengua escrita en relación con la multiplicidad de usos sociales en los que la misma está inmersa. Alfabetización en un espacio y tiempo histórico, geográfico, político y social determinado. Estar alfabetizados como la posibilidad de los sujetos de ser lectores y productores de la lengua escrita en un proceso que comienza con el nacimiento y continúa a lo largo de toda la vida. En este sentido, acordamos con Zamero en que la alfabetización implica un proceso de naturaleza lingüística que consiste en el aprendizaje de una lengua escrita, que conlleva el desarrollo de procesos cognitivos de lectura y escritura y de prácticas culturales que los constituyen (Zamero et al., 2009). La alfabetización es un proceso que involucra un sistema de valoraciones al interior de la comunidad que se alfabetiza (Zamero, 2006). En palabras de Bautier y Bucheton (1997), podemos pensar en la alfabetización como apropiación de un lenguaje en tanto actividad lingüística, social, cognitiva y subjetiva; como proceso heterogéneo atravesado por dimensiones culturales, sociales y lingüísticas a la vez singulares (propias del sujeto que las produce) y compartidas (por grupos que elaboran formas y normas y las reconocen como propias). Lejos de ser habilidades o estrategias neutras, dichos autores sostienen que la lectura y la escritura son prácticas del lenguaje, históricas, culturales, identitarias, lingüísticas y cognitivas; adquiridas por participación en las comunidades 
en las que se ejercen esas prácticas. Son un instrumento de poder para el sujeto, ya que le permiten adquirir mayor dominio del mundo y de sí mismo. Las lecturas y escrituras dependen de las evoluciones tecnológicas, las conductas lingüísticas de una población, sus rituales, usos y costumbres. La alfabetización involucra, en forma simultánea, la apropiación de conocimientos sobre el sistema de escritura, los géneros y el lenguaje escrito, las prácticas de lectura y escritura que circulan socialmente, todo a la vez.

Freire y Ferreiro han contribuido enormemente al campo educativo y de transformación política desde lugares diferentes, en tiempos y espacios geográficos propios. Desde nuestro punto de vista, ambos trabajos son convergentes y complementarios: la didáctica constructivista con foco de enseñanza en las prácticas sociales del lenguaje, sobre la base de las investigaciones psicogenéticas, es la práctica alfabetizadora más consistente con los postulados de la educación popular freireana que defiende y materializa la alfabetización como acto de creación, diálogo, reflexión, análisis crítico y contextualización de los procesos de aprendizaje. En este sentido, promueve verdaderos procesos emancipatorios al postular la construcción de personas lectoras y escritoras críticas y autónomas a lo largo de la vida. Consideramos que el enfoque constructivista implica una concepción didáctica superadora del método freireano de alfabetización y más consecuente aun con sus postulados de educación liberadora, su pedagogía de la esperanza y de la emancipación.

En este sentido, listamos algunas diferencias entre el método de alfabetización freireano y el enfoque constructivista:

" La idea de "método" se asocia a pasos predeterminados e iguales para toda persona que se alfabetiza, a diferencia de un "enfoque" que postula una mirada sobre el sujeto que aprende a leer y escribir, e intervenciones posibles y diversas que acompañen su camino de escritura singular.

»Pensar una etapa de "alfabetización" y otra de "postalfabetización" es diferente a pensar procesos continuos de alfabetización a lo largo de toda la vida, que implican diversos soportes, ámbitos y géneros, en función de prácticas sociales y culturales concretas, y que retoman lo que las personas piensan e hipotetizan acerca del sistema de escritura, más allá de haber ido o no a la escuela.

» Partir de una misma palabra para todo el grupo que se alfabetiza, como "palabra generadora" (aquella que, descompuesta en sus elementos silábicos, propicia, por la combinación de esos elementos, la creación de nuevas palabras) que va a descomponerse en familias de sílabas y luego a reagruparse en la producción de palabras nuevas con combinaciones posibles, además de producir escrituras sin sentido social y comunicativo, impone escrituras alfabéticas sin tomar en cuenta procesos de escritura presilábicos, silábicos o silábico-alfabéticos propios de las personas que aprenden a leer y escribir. Es muy diferente a pensar en la construcción de un ambiente alfabetizador, con presencia de palabras diversas, conocidas, seguras, que son parte del universo vocabular y que están al servicio de la problematización, análisis, comparación e interpretación para la producción de nuevas escrituras; así como también pensar en la reflexión sobre las relaciones entre oralidad y escritura, desde el análisis de las partes y el todo, y desde intervenciones de enseñanza que acompañen a pensar sobre dichas relaciones.

El enfoque didáctico-constructivista con base en las investigaciones psicogenéticas y didácticas es totalmente consecuente - sin proponérselo - con la idea freireana de que "alfabetizar es ante todo abrir espacios de lucha, en donde los oprimidos se transformen en sujetos en un proceso de conocimiento transformador de su realidad" (Varela Barraza y Escobar Guerrero, en Freire 1984: 12). Nuestras propias investigaciones aportan evidencia acerca del cambio que se produce en las personas, en la visión de sí mismas como lectoras, escritoras y aprendices, al avanzar en sus procesos de alfabetización, transformándose visiones desvalorizadoras y culpabilizadoras, en la posibilidad 
de objetivar la propia realidad desde una comprensión de vulneración de los propios derechos por parte de la sociedad y demandar más y mejor educación, salud, trabajo, vivienda, a lo largo de sus vidas.

Aunque el mismo Freire (1984) haya planteado que siempre vio a la alfabetización de adultos como un acto político y un acto de conocimiento, y por eso mismo como un acto creador, y que para él sería imposible comprometerse en un trabajo de memorización mecánica de ba-be-bi-bo-bu, de la-le-li-lo-lu o reducir la alfabetización a la pura enseñanza de la palabra, de las sílabas o de las letras, desde nuestro punto de vista la codificación y descodificación poseen limitaciones y obstáculos a los actos creativos y constructivos sobre el sistema de escritura y el lenguaje que se escribe. También afirmó su descreimiento de las cartillas "que presentan los signos gráficos como una donación y reducen al analfabeto más a una condición de objeto de su alfabetización que de sujeto de la misma" (Freire, 2004: 72). Sin embargo, usualmente las prácticas o campañas alfabetizadoras que retoman sus postulados suelen basarse en cartillas predeterminadas. Desde el enfoque constructivista defendemos proyectos didácticos con función social y comunicativa, que se originen en los intereses y necesidades de los grupos que se alfabetizan y que puedan contemplar las situaciones didácticas fundamentales que ya hemos descrito, así como planificar intervenciones didácticas específicas según los caminos de escritura diversos en el grupo. Ello condice con el pensamiento freireano:

(...) la alfabetización es más que el simple dominio psicológico y mecánico de las técnicas de escribir y leer. Es el dominio de estas técnicas en términos conscientes. Es entender lo que se lee y escribir lo que se entiende. Es comunicarse gráficamente. Es una incorporación. Implica no una memorización visual y mecánica de cláusulas, de palabras, de sílabas, incongruentes con el universo existencial — cosas muertas o semimuertas, sino una actitud de creación y recreación. (Freire, 2004: 72)

Y agregamos: construcción y reconstrucción del sistema de escritura como sistema de representación, no como una técnica de transcripción, como nos ha enseñado Ferreiro.

En el capítulo "El pueblo dice su palabra o la alfabetización en São Tomé e Príncipe" (Freire, 1984), se lee que Freire una vez más se coloca frente a las páginas en blanco para escribir sobre el proceso de alfabetización de adultos, con el espíritu de quien discute un tema por primera vez. Siempre como asunto inconcluso, piensa y repiensa el proceso de alfabetización como quien se encuentra frente a una novedad, desde su propia práctica. El espíritu de este artículo ha sido alimentar y actualizar estos pensamientos, a modo de diálogo en nuevos tiempos y lugares. En La importancia de leer y el proceso de liberación (1984), Freire da ejemplos del "Cuaderno de ejercicios: Practicar para aprender" utilizado en la denominada fase de alfabetización y del "Cuaderno de Cultura Popular", utilizado en la fase de posalfabetización. Allí, en sucesivas codificaciones (fotografías) que ilustran jóvenes nadando y jóvenes trabajando, dice: "Es nadando como se aprende a nadar", "Es trabajando como se aprende a trabajar", y al pie de página: "Practicando aprendemos a practicar mejor" (1984: 136-137). En consonancia con estas frases (aunque sin conocerlas antes), decimos siempre, desde el enfoque que defendemos, que "a leer y escribir se aprende leyendo y escribiendo. Y mucho".

A leer y escribir se aprende leyendo y escribiendo en interacción continua y progresiva con multiplicidad de textos de circulación social. Pensamos la alfabetización considerando a los sujetos escritores y lectores plenos aunque aún no sepan leer y escribir convencionalmente. Apostamos a un trabajo en el que se acepte todo el proceso de construcción, desde el mismo momento en que se percibe que el sujeto ya se está interrogando sobre el sistema. Aquí nacen el diálogo, la problematización, la toma de conciencia, los actos creadores, la reconstrucción de la cultura, la transformación personal y colectiva, la esperanza y la liberación. 


\section{Q Bibliografía}

"Bautier, E. y Bucheton, D. (1997). Les pratiques socio-langagières dans la classe de français? Quels enjeux? Quelles démarches? (¿Las prácticas sociales del lenguaje en la clase de francés? ¿Qué desafíos? ¿Cuáles procesos?). Repères. Institut National de Recherche Pédagogique, 15(13), 11-25. Perelman, F. (trad.).

"Castedo, M. (2010). Voces sobre la alfabetización inicial en América Latina 19802010. Revista Lectura y Vida: 35-68, diciembre.

»Castedo, M.; Siro, A. y Molinari, C. (2009). Enseñar y aprender a leer. Buenos Aires, Novedades Educativas.

» Ferreiro, E. et al. (1983). Los adultos no alfabetizados y sus conceptualizaciones del sistema de escritura. Departamento de Investigaciones Educativas. Cuaderno de Investigaciones educativas, № 10. México.

» Ferreiro, E. et al. (1986). Proceso de alfabetización. La alfabetización en proceso. Buenos Aires, Centro Editor de América Latina.

» Ferreiro, E. et al. (1987). Alternativas a la comprensión del analfabetismo en la región. Boletín Proyecto Principal de Educación en América Latina y el Caribe, № 13. Santiago de Chile, Unesco.

» Ferreiro, E. et al. (1994). Las condiciones de alfabetización en medio rural. México, DIECINVESTAV.

» Ferreiro, E. et al. (1999). Cultura escrita y educación. México, Fondo de Cultura Económica.

» Ferreiro, E. y Gómez Palacio, M. (1982). Análisis de las Perturbaciones en el proceso de aprendizaje de la lecto-escritura. México, Dirección General de Educación Especial (5 fascículos).

» Ferreiro, E. y Teberosky A. (1979). Los sistemas de escritura en el desarrollo del niño. México, Siglo XXI.

» Ferreiro, E. y Zamudio, C. (2008). La escritura de sílabas CVC y CCV en los inicios de la alfabetización escolar. ¿Es la omisión de consonantes prueba de la incapacidad para analizar la secuencia fónica? RIPLA VIII, 1-2, pp. 37-55. Pisa, Roma, Fabrizio Serra Editore.

» Freire, P. (1970). Pedagogía del oprimido. México, Siglo XXI.

»Freire, P. (1976). Educación y cambio. Buenos Aires, Galerna.

» Freire, P. (1984). La importancia de leer y el proceso de liberación. México, Siglo XXI.

»Freire, P. (1998). La importancia del acto de leer y el acto de liberación. México, Siglo $\mathrm{XXI}$.

»Freire, P. (2004). La educación como práctica de la libertad. Buenos Aires, Tierra del Sur.

»Freire, P. y Macedo, D. (1989). Alfabetización. Lectura de la palabra y lectura de la realidad. Barcelona, Paidós.

»Grunfeld, D. (2012). La palabra escrita y la palabra oral al final de sala de 5 años. Contraste entre dos propuestas de enseñanza en escuelas públicas. Tesis de Maestría en Escritura y Alfabetización. Facultad de Humanidades y Ciencias de 
la Educación, Universidad Nacional de La Plata. Directora: Dra. Emilia Ferreiro. Codirectora: Mg. Claudia Molinari. (inédita).

》 Kaufman, A. M. (1988). La lecto-escritura y la escuela. Buenos Aires, Santillana.

» Kurlat, M. (2011). Procesos de alfabetización en jóvenes y adultos: Ismael y sus laberintos de escritura. Revista Interamericana de Educación de Adultos, año 33, № 2: 69-95. México, CREFAL.

» Kurlat, M. (2014a). Procesos de alfabetización inicial en personas jóvenes y adultas: entre el mundo de la vida y el mundo de la escuela. En Castorina, J.y Orce, V. (comps.). Perspectivas en la investigación educativa: Contribuciones de los/las Investigadores/as en formación. Buenos Aires, Secretaría de Publicaciones de la Facultad de Filosofía y Letras, UBA, Investigadores en Formación del Instituto de Investigaciones en Ciencias de la Educación.

» Kurlat, M. (2014b). La historia de Gabriel, entre el mundo de la escuela y el mundo de los diarios. Revista Decisio, Centro de Cooperación Regional para la Educación de Adultos en América Latina y el Caribe, № 37, sección Testimonios, enero-abril.

»Kurlat, M. (2014C). El „“culto a las letras” en los procesos de alfabetización inicial de personas jóvenes y adultas: un obstáculo en los caminos de escritura. Revista Interamericana de Educación de Adultos, año 36, № 1: 59-90, enero/junio. México, CREFAL.

"Kurlat, M. (2014d). Procesos de lectura y escritura en personas jóvenes y adultas: caminos hacia otros mundos posibles. Revista Decisio, Centro de Cooperación Regional para la Educación de Adultos en América Latina y el Caribe, № 37: 3-10, enero-abril.

» Kurlat, M. (2016). Procesos psicosociales y didácticos en la alfabetización inicial de personas jóvenes y adultas: la urdimbre y la trama. Revista Veras, revista acadêmica de Educação do Instituto Vera Cruz, São Paulo, vol. 6, № 1: 69-86, janeiro/junho.

» Kurlat, M. y Chichizola, D. (2017). Enseñar a leer y escribir en las aulas de jóvenes y adultos: un diálogo entre docencia e investigación en un proceso colectivo de construcción de conocimientos. Revista Interamericana de Educación de Adultos, № 40, enero-junio, CREFAL, México.

» Kurlat, M.y Perelman, F. (2012). Procesos de alfabetización inicial en personas jóvenes y adultas ¿Hacia una historia de inclusión? Revista del Instituto de Investigaciones en Ciencias de la Educación, № 32: 55-72 Buenos Aires, Editorial de la Facultad de Filosofía y Letras UBA.

"Molinari, C. (2007). Estabilidad y variación de las palabras en los inicios de la alfabetización (escritura manual y a computadora). Tesis de Maestría en Ciencias en la especialidad de investigaciones educativas. Dirección: Dra. Emilia Ferreiro, Centro de Investigación y de Estudios avanzados del Instituto Politécnico Nacional.

" Molinari, C. y Ferreiro, E. (2007). Identidades y diferencias en la escritura en papel y en computadora en las primeras etapas del proceso de alfabetización. Lectura y Vida, 28, 4 .

»Lorenzatti, M. C. (2009). Conocimientos, prácticas sociales y usos escolares de cultura escrita de adultos de baja escolaridad, Universidad Nacional de Córdoba, Tesis de Doctorado (inédita).

"Quinteros Sciurano, G. (2004). La libertad de las letras. En Pellicer, A. y Vernon, S. (coords.). Aprender y enseñar la lengua escrita en el aula. México, SM.

"Quinteros Sciurano, G. (1997). El uso y función de las letras en el período prealfabético. Tesis de Doctorado. Tesis 27. México, DIE-CINVESTAV. 
» Teberosky, A. (1982). Construcción de escrituras a través de la interacción grupal. En Ferreiro, E. y Gómez Palacios, M. (comps.). Nuevas perspectivas sobre la lectura y la escritura. México, Siglo XXI.

»Torres, R. M. (2008). Alfabetización y acceso a la cultura escrita por parte de jóvenes y adultos excluidos del sistema escolar. Un estudio de campo en 9 países de América Latina y el Caribe. CREFAL-Fronesis.

»Toubes, A.; Buitron, V.; Cruciani, S.; Kaufmann, C.; Kurlat Marucco, M.; Ronzoni, Serrano M.; Topasso, P. y Vignau, S. (2008). Procesos de enseñanza y aprendizaje de la lectura y la escritura en el aula de jóvenes y adultos. Estudio comparativo de casos. Revista Interamericana de Educación de Adultos, № 2 , año 30: 73-94. México, CREFAL.

»Vernon, S. (1991). El proceso de construcción de la correspondencia sonora en la escritura (en la transición entre los períodos presilábico y silábico). Tesis 6, México, DIE-CINVESTAV.

»Vernon, S. (1997). Escritura y conciencia fonológica en niños hispano-parlantes. Infancia y Aprendizaje, № 81: 105-120.

»Vernon, S. (1999). Letras y sonidos en la alfabetización inicial. Cuadernos de Trabajo, Consejo Nacional de Ciencia y Tecnología. México.

"Vernon, S. (2004). ¿Qué tanto es un pedacito? El análisis que los niños hacen de las partes de la palabra. En Pellicer, A. y Vernon, S. (coords.). Aprender y enseñar la lengua escrita en el aula. México, SM.

»Zamero, M.; Taboada, M. B.; Cicarelli, M. y Rodríguez, M. (2009). Algo más que las primeras letras: alfabetización inicial. En La formación en investigación inicial de los futuros docentes (versión preliminar, no publicada).

»Zamudio, C. (2004). Las ideas infantiles sobre la separación de las palabras. En Pellicer, A. y Vernon, S. (coords.). Aprender y enseñar la lengua escrita en el aula. México, SM.

"Zamudio, C. (2008). Influencia de la escritura alfabética en la segmentación de sonidos vocálicos y consonánticos. Revista Lectura y Vida, 29, 01: 10-21, marzo.

"Zamudio, C. (2004). Las ideas infantiles sobre la separación de las palabras. En Pellicer, A. y Vernon, S. (coords.). Aprender y enseñar la lengua escrita en el aula. México, SM.

\section{Marcela Kurlat}

Doctora en Educación por la Universidad de Buenos Aires. Magíster en Psicología Educacional, Facultad de Psicología (UBA). Licenciada en Ciencias de la Educación (UBA). Docente e Investigadora del Programa de Desarrollo Sociocultural y Educación Permanente: la educación de jóvenes y adultos más allá de la escuela (Instituto de Investigaciones en Ciencias de la Educación, Universidad de Buenos Aires). marcelakurlat@yahoo.com.ar 